\title{
Наблюдения искусственных космических объектов в Крымской астрофизической обсерватории на зеркальном телескопе им. акад. Г.А. Шайна
}

\author{
В.В. Румянцев, В.В. Бирюков \\ ФГБУН “Крымская астрофизическая обсерватория РАН", Научный, 298409, Крым \\ rum@craocrimea.ru
}

Поступила в редакцию 19 июля 2021 г.

\begin{abstract}
Аннотация. В статье приводится обзор исследований искусственных объектов околоземного космического пространства, проводимых на ЗТШ с 2005 г. по настоящее время. Одна из целей исследования состояла в отработке методики наблюдений и каталогизации малоразмерных объектов $(\sim 10-$ 25 см) с блеском 18-20 наблюдательного времени, использование ЗТШ для решения данной задачи было достаточно эффективно. Показано, что в геостационарной области возможно успешно проводить обнаружение и каталогизацию малоразмерных объектов даже с телескопом с малым полем зрения ( 8-12 угл. мин.).

Для решения задач поиска слабых, сильно переменных, “динамичных" объектов был создан новый современный прибор - панорамный ПЗС-фотометр, размещаемый в прямом фокусе 3ТШ.

С 2011 по 2021 г. на ЗТШ проводились наблюдения далеких KA "Спектр-Р", Gaia, "Спектр-РГ" и Mars-2020. Особое внимание уделено российской астрофизической обсерватории “Спектр-РГ", находящейся на галоцентрической орбите вокруг точки Лагранжа L2 системы Солнце-Земля. Наблюдения за этим научным аппаратом продолжаются до сих пор. Точность получаемых астрометрических оценок такова, что медианные среднеквадратичные ошибки положения составляют $0.055^{\prime \prime}$ и $0.075^{\prime \prime}$ по RA и Decl соответственно. KA Mars-2020, находясь на траектории полета к Марсу, наблюдался на расстоянии вплоть до 6.5 млн км как объект $21.8^{m}$.

Задача наблюдений далеких КА остается актуальной для распознавания искусственных объектов среди многочисленных естественных, открываемых в ближнем околоземном пространстве.
\end{abstract}

Ключевые слова: околоземное пространство, геостационарная область, искусственные космические объекты, малоразмерный космический мусор, далекие космические аппараты

\section{1 Введение}

Наблюдения искусственных космических объектов в КрАО на ЗТШ можно разделить на два этапа.

Первый этап проходил с 1962 по 1973 год. Он был связан с наблюдениями первых далеких космических аппаратов, запускаемых к Луне и Марсу. В то время радиотехнических средств контроля положения космических аппаратов (KA) в пространстве попросту не было, и поэтому для указанной цели использовались прямые оптические наблюдения далеких КА. Эта часть истории ЗТШ достаточно подробно изложена в ряде публикаций (см., например, Прокофьева-Михайловская, 2008). Стоит отметить, что за 11 лет были проведены наблюдения 19 аппаратов, из них 14, запущенных к Луне, включая пролетные траектории, и 5 - к Марсу.

Второй этап наблюдений объектов в околоземном космическом пространстве был связан с исследованиями малоразмерного "космического мусора" в геостационарной области (с 2005 по 2012 г.), а также наблюдениями избранных далеких космических аппаратов, которые проводятся и в настоящее время.

В 2003 году сотрудники Института прикладной математики им. М.В. Келдыша (ИПМ) В.М. Агапов и И.Е. Молотов обратились к нам с просьбой попытаться найти и пронаблюдать малоразмерные 
объекты в геостационарной области так, чтоб была возможность построить по ним надежную орбиту для последующей каталогизации. Под малоразмерными здесь подразумеваются слабые объекты с блеском слабее $16^{m}$, что на геостационарной орбите (ГСО) соответствует размерам менее 1 метра.

В то время за рубежом уже проводились наблюдения малоразмерного космического мусора, но эти работы имели в основном лишь статистический характер. Для страховки новых космических аппаратов необходимо было оценивать их риски столкновения с космическим мусором. С этой целью создавались теоретические модели "засоренности" околоземного пространства, а уже для верификации этих моделей проводили соответствующие наблюдения. В ходе данной работы подсчитывались все объекты, проходящие по полю зрения неподвижного телескопа. Размер объекта оценивался по блеску и среднему альбедо. Такие "верификационные" наблюдения в Европе и США проводились эпизодически. Получаемые в них результаты вносили важный, но сильно ограниченный вклад в изучение космического мусора. О каталогизации таких объектов речи не было.

До недавнего времени (начало 2000-х годов) исследование засорения ГСО основывалось в основном на регулярных обновлениях орбитальных данных, произведенных и распространяемых Сетью надзора космического пространства США (US Space Surveillance Network), а также на наблюдениях, полученных в рамках согласованных наблюдательных кампаний под эгидой Межведомственного координационного комитета по космическому мусору (IADC) (Technical Report on Space Debris, 1999). Эти наблюдения показали присутствие в области ГСО значительного количества объектов различной яркости $15-20^{m}$ (и, соответственно, различных размеров). При данной методике наблюдений не было возможности построить надежные орбиты зарегистрированных объектов (Klinkrad et al., 2005; Seitzer et al., 2005; Schildknecht et al., 2001).

Активные наблюдения малоразмерного космического мусора также проводились на 1-м Цейссе в обсерватории Teide на о. Тенерифе (Schildknecht et al., 2005). Они показали большое количество некаталогизированных объектов в геостационарной области. Однако сами исследования, как и описанные выше, носили больше статистический характер и не позволяли отслеживать обнаруженные фрагменты длительное время. Таким образом, невозможно было рассчитать точные орбиты и каталогизировать объекты (Schildknecht et al., 2005).

Значительную долю среди наблюдаемых объектов составляли фрагменты разрушений ИСЗ. То, что искусственные объекты в геостационарной области разрушаются, косвенно проявлялось в эпизодически обнаруживаемых значимых спорадических изменениях их орбитальных элементов. Медленные изменения элементов орбит со временем вдруг резко меняются, и это явный признак того, что с объектом что-то произошло (Sochilina et al., 2001, 2002). K концу 90-х годов были известны несколько случаев разрушения объектов в геостационарной области. Однако при этом местоположения и пространственные распределения фрагментов оставались неизвестными. Поиск этих фрагментов и исследование населенности разрушенных объектов имели важное значение для оценки риска столкновения с ними.

С этой целью в 2003 году на частично автоматизированном телескопе АТ-64 (D = 640 мм, F/1.4) начались пробные наблюдения. Они проводились как по самостоятельным программам поиска, так и совместно в рамках наблюдательных кампаний IADC. В результате этих исследований стали обнаруживаться новые объекты ближнего космоса, не входящие в публичные каталоги более развитых американских средств наблюдений за OKП (Agapov et al., 2005a, b). Их блеск достигал 17-18 а астрометрическая точность измерения была лучше $1^{\prime \prime}$, что раньше было не достижимо на отечественных средствах наблюдения. Первый объект под номером 90003 был закаталогизирован 18 октября 2004 г.

В отдельных случаях попадались объекты с сильно переменным блеском, таким, что в минимуме они не регистрировались 64-см телескопом. Кроме того, слабые объекты на больших фазовых углах еще больше теряли свой блеск, что ограничивало их мерную дугу. И самое главное - был большой интерес посмотреть населенность околоземного пространства объектами слабее $18^{m}$. Это обстоятельство потребовало привлечения более крупных телескопов. Для этой цели в 2003 году были возобновлены наблюдения в прямом фокусе ЗТШ - самом крупном телескопе КрАО. Такого типа наблюдения не проводились на нем с 1963 г. По сути, потребовалась полная переделка кассеты прямого фокуса, поскольку все предыдущие варианты предназначались для использования фотографических пластинок в качестве детектора. Имевшиеся корректоры поля были рассчитаны для различных спектральных полос и на очень короткий задний отрезок. Изготовление современ- 


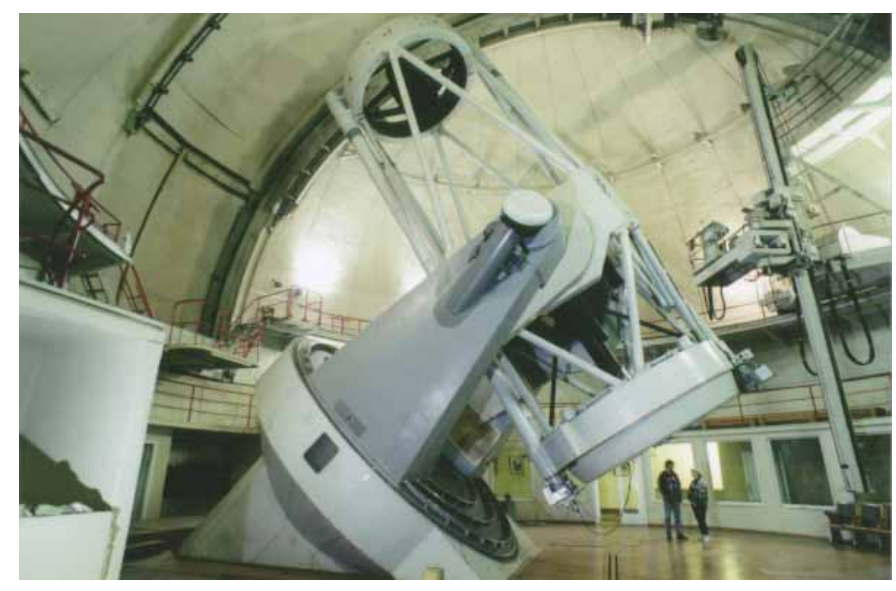

ЗТШ, прямой фокус

$\mathrm{F}=10000 \mathrm{~mm}$

$\mathrm{D}=2600 \mathrm{~mm}$

CCD: FLI PL-1001E, $1024 \times 1024$ @ $24 \mathrm{mcm}$

Scale: $0.5 \mathrm{arcsec} / \mathrm{pix}$

FOV: $8.4 \times 8.4$ arcmin

Limit mag GEO: $20 \mathrm{~m} @ 1 \mathrm{sec}$

Рис. 1. Зеркальный телескоп им. академика Г.А. Шайна

ного фотометра прямого фокуса с ПЗС-детектором дало новую жизнь панорамным наблюдениям на ЗТШ.

Следует отметить, что ЗТШ, в используемой нами конфигурации, имеет фокусное расстояние, равное 10 м. В то время мы использовали в качестве детектора ПЗС-камеру FLI IMG-1001Е, выделенную нам сотрудниками ИПМ, что давало поле зрения всего $8.4 \times 8.4$ угл. мин. (рис. 1). Такое поле зрения очень мало для поисковых работ. Тем не менее была разработана и апробирована методика наблюдений и поиска предельно слабых объектов на ГСО.
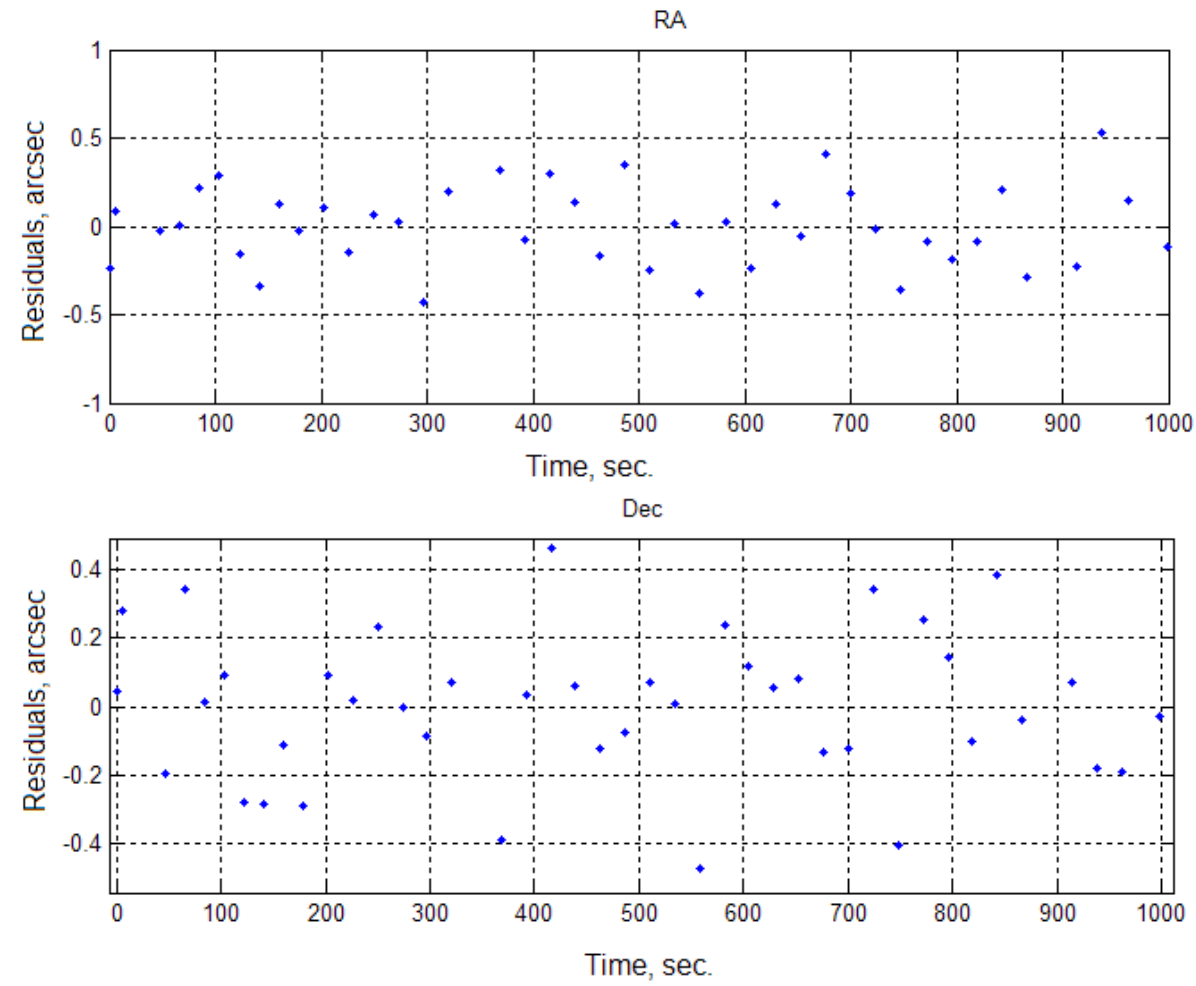

Рис. 2. Невязки положений объекта 90024, наблюдаемого на ЗТШ 19 сентября 2006 г.

В результате комплекса проведенных работ было обнаружено значительное количество слабых фрагментов до $20^{m}$, что на расстоянии геостационарной области соответствует размеру в 10 см! 
Длинный фокус ЗТШ также позволил увеличить точность наблюдаемых слабых объектов. В качестве примера на рисунке 2 показаны невязки объекта 90024, наблюдаемого на ЗТШ 19 сентября 2006 г. Среднеквадратичные ошибки положения составляли по RA - 0.23", по Decl. $-0.22^{\prime \prime}$.

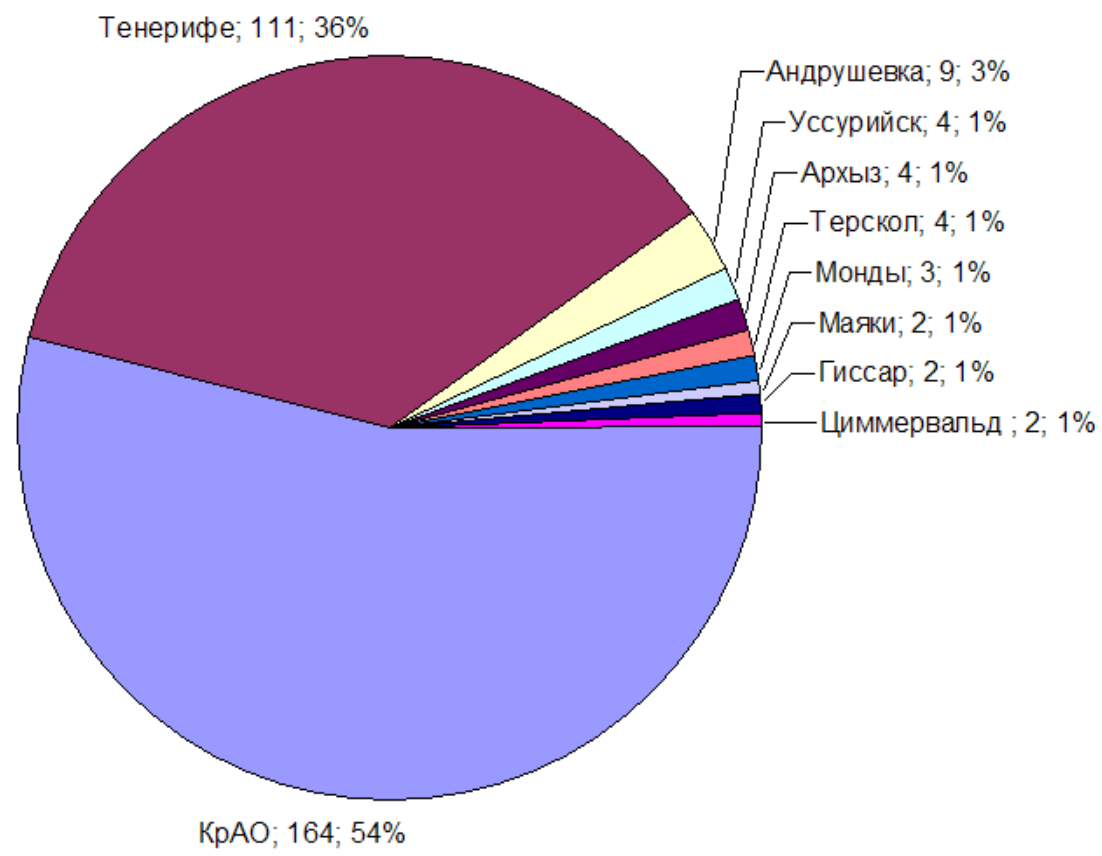

Рис. 3. Распределение каталогизированных малоразмерных объектов по обсерваториям в период 2007-2009 гг

ЗТШ - прежде всего астрофизический телескоп, используемый сотрудниками КрАО для спектральных и поляриметрических наблюдений звезд и галактик, и для наших задач на нем выделялось не более 3-х ночей в месяц (благодарность Р.Е. Гершбергу - на тот момент зав. лабораторией физики звезд и галактик, активно поддержавшему данный проект). Тем не менее в то время темпы открытия новых малоразмерных объектов в месяц на нем были выше, чем на любом другом телескопе (рис. 3 ). Это, в свою очередь, породило еще одну проблему. Открытые на ЗТШ слабые объекты больше не на чем было наблюдать, а следующий сеанс наблюдений наступал только через месяц. Это приводило к потере объектов. Для преодоления проблемы были ужесточены требования на длину мерной дуги открываемых объектов. Вдобавок мы стали прорабатывать методику поиска объекта вдоль траектории на телескопах с малым полем зрения. Предпринимаемые действия позволяли находить некоторые объекты даже через месяц после их первоначального обнаружения. Помимо этого, в 2007 году для поддержки наблюдений слабых объектов был привлечен телескоп с апертурой 1 метр Цейсс-1000 на горе Кошка недалеко от Симеиза. Во время вынужденного простоя, из-за ограничения наблюдательного времени, оборудование прямого фокуса ЗТШ вывозилось в Симеиз, где в течение нескольких ночей проводились наблюдения, после чего аппаратура возвращалась обратно. Наблюдения на метровом Цейссе отчасти были полезны для поддержания актуальности орбит слабых объектов, что позволило сократить количество потерь новых объектов, обнаруженных на ЗТШ, но полноценно заменить ЗТШ он не мог.

Через год таких наблюдений стало понятно, почему Г.А. Шайн инициировал после войны перенос обсерватории из Симеиза в нынешнее расположение - пос. Научный. Из-за близости к южной гряде Крымских гор с одной стороны и к морю с другой создавались неблагоприятные астроклиматические условия для наблюдений. Более того, "расцвет дикого капитализма" на южном берегу Крыма в начале 2000-х годов и незащищенность астрономических учреждений от излишней засветки (что продолжается до сих пор) лишь усугубляли ситуацию. После двух лет активной оптической поддержки наблюдения космического мусора в Симеизе были прекращены и переведены в другое место. 


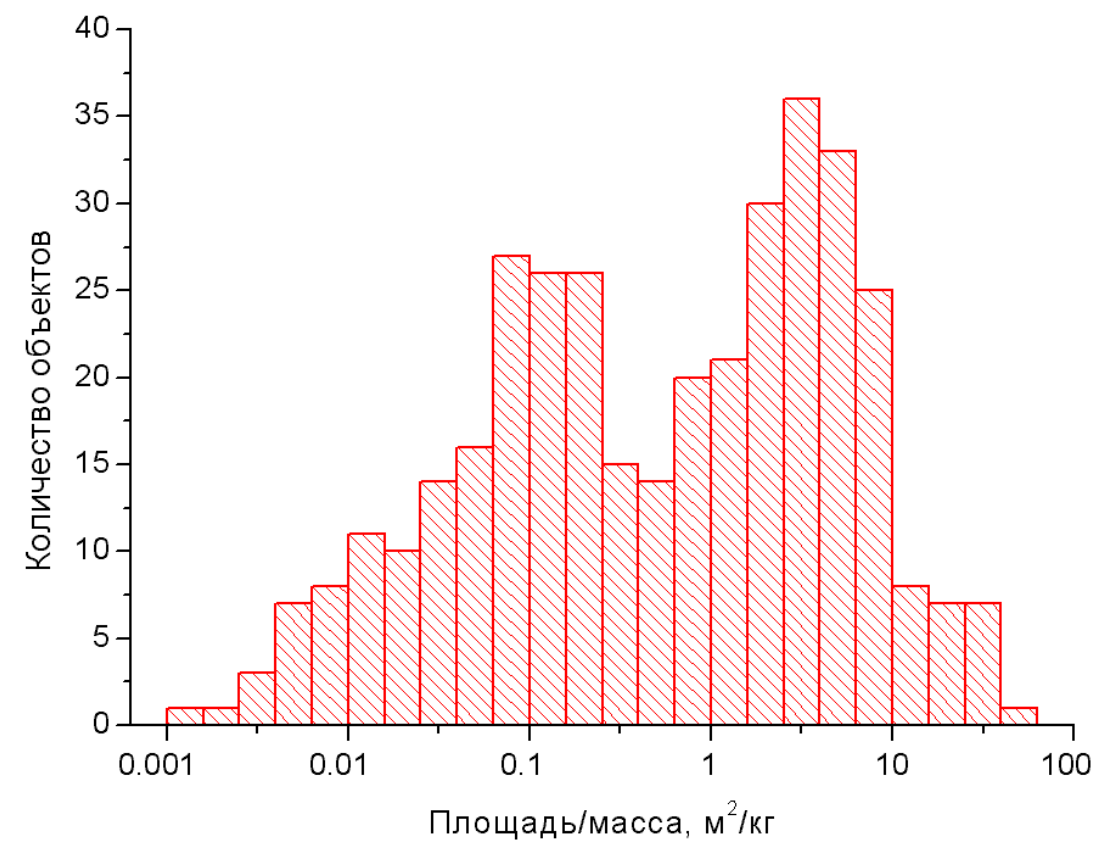

Рис. 4. Распределение отношения площади поперечного сечения к массе для 367 объектов на конец 2011 г.

При тесном взаимодействии с сотрудниками Крымской лаборатории ГАИШ МГУ частично были задействованы инструменты Южной станции Цейсс-600 и ЗТЭ с апертурами 60 и 125 сантиметров соответственно. Наблюдения на этих телескопах были не столь интенсивными, однако они оказались полезными при отработке методики наблюдений, а также позволили обнаружить и закаталогизировать несколько достаточно слабых объектов в области ГСО.

В итоге темпы открытий были настолько высоки, что КрАО в то время занимала лидирующие позиции среди обсерваторий, вовлеченных в описываемые исследования.

Одним из значимых достижений первых лет сотрудничества наблюдателей и баллистиков было подтверждение существования "облаков" фрагментов в области ГСО, порожденных разрушением космических аппаратов "Экран” и ступеней "Транстейдж”. Еще более значимым результатом было независимое подтверждение существования малоразмерных объектов с таким большим отношением площади к массе (AMR), что влияние светового давления приводит к существенной эволюции их орбит и делает невозможным долговременный прогноз траектории. На данный момент обнаружены объекты с отношением площади к массе до $70 \mathrm{~m}^{2} /$ кг, которые в буквальном смысле "сметаются с орбиты" солнечным излучением (рис. 4). Отметим, что типовые значения AMR для космических аппаратов лежат в диапазоне $10^{-3}-10^{-2} \mathrm{M}^{2} /$ кг. Благодаря интенсивным наблюдениям и качественным баллистическим расчетам такие объекты стали сопровождать, не теряя в течение длительного времени.

\section{2 Наблюдения далеких КА}

Оптические наблюдения далеких космических аппаратов также остаются востребованными и в 21 веке. С 2011 по 2021 гг. на ЗТШ проводились наблюдения KA "Спектр-Р", Gaia, "Спектр-РГ" и Mars-2020. Среди них достойны внимания следующие:

\section{Gaia (2013-074A)}

В конце 2014 года в рамках работ по оценке возможности привлечения наземных оптических обсерваторий для наблюдения КА “Спектр-РГ" на траектории перелета и в окрестности либрационной точки L2 системы Солнце- Земля в KрAО РАН и ИСЗФ СО РАН были проведены тестовые наблюдения KA Gaia. 
KA Gaia - космический телескоп оптического диапазона, разработанный Европейским космическим агентством, был запущен в окрестность точки L2 19 декабря 2013 г., а 8 января он вышел на галоцентрическую орбиту вокруг L2.

На ЗТШ 26 октября 2014 были проведены 2 сета наблюдений KA Gaia (с 21:05:11 по 23:39:58), всего получено 26 положений. В выделенное время погода была не очень благоприятной, поэтому количество наблюдений было ограничено. Объект имел блеск 20.2-21.2 ${ }^{m}$. Среднеквадратичные ошибки невязок составили 0.35 и $0.36^{\prime \prime}$ (Захваткин, 2015). Ниже представлена сравнительная таблица 1 точности наблюдений на двух телескопах.

Таблица 1.

\begin{tabular}{lcccc}
\hline Телескоп & Интервал наблюдений & $\begin{array}{c}\text { кол-во } \\
\text { положений }\end{array}$ & $\begin{array}{c}\text { CKO RA } \\
\text { (угл. сек. })\end{array}$ & $\begin{array}{c}\text { CKO Decl } \\
\text { (угл. сек.) }\end{array}$ \\
\hline АЗТ-33ИК, 1.5 м & $\begin{array}{c}\text { 24 сентября - } \\
\text { 1 октября 2014 }\end{array}$ & 45 & 0.56 & 0.73 \\
ИСЗФ СО РАН & 26 октября 2014 & 26 & 0.35 & 0.36 \\
\hline $\begin{array}{l}\text { ЗТШ, 2.6 м } \\
\text { КрАО РАН }\end{array}$ & & & 0.77 & 0.34 \\
\hline $\begin{array}{l}\text { АТ-3ЗИК, 1.5 м } \\
\text { ИСЗФ СО РАН }\end{array}$ & 12-26 ноября 2014 & 35 & & \\
\hline
\end{tabular}

\section{“Спектр-РГ" (2019-040A)}

13 июля 2019 года с космодрома Байконур была запущена рентгеновская обсерватория "Спектр-РГ" (44432), предназначенная для построения полной карты видимой части Вселенной в рентгеновском диапазоне излучения. Обсерватория выводилась на гало-орбиту вокруг точки Лагранжа L2, находящейся на расстоянии 1.5 млн км от Земли (рис. 5).

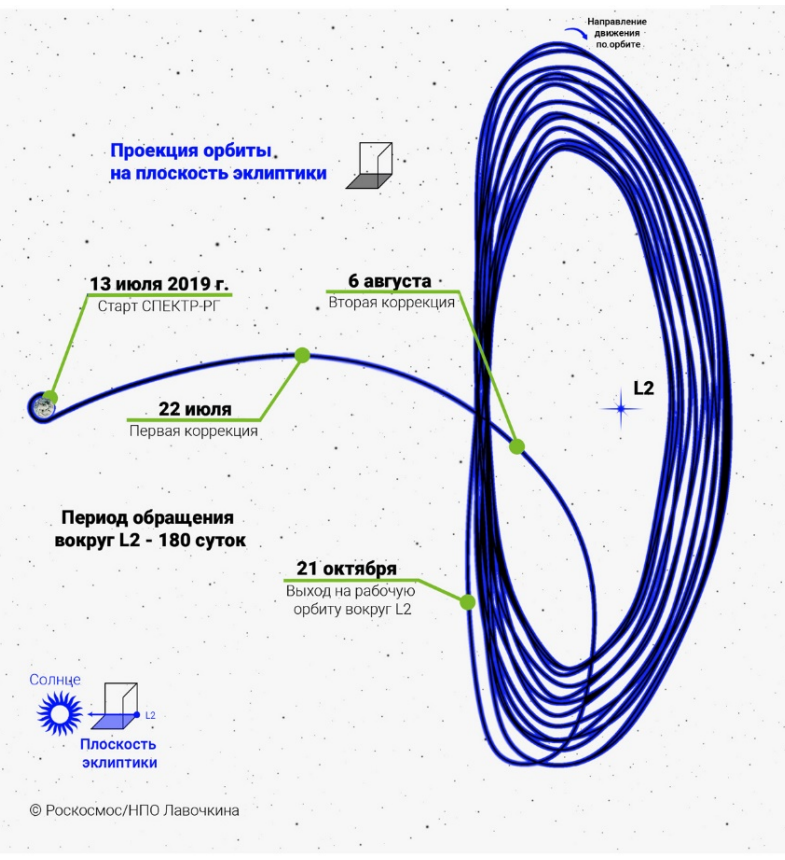

Рис. 5. Траектория выведения рентгеновской обсерватории “Спектр-РГ" в точку L2 ((c) Роскосмос)

Астрометрические наблюдения КА “Спектр-РГ" проводились по просьбе наших коллег из ИПМ РАН и ИКИ РАН и имели цели уточнения траектории аппарата и минимизации расхода топлива при плановых коррекциях орбиты. 

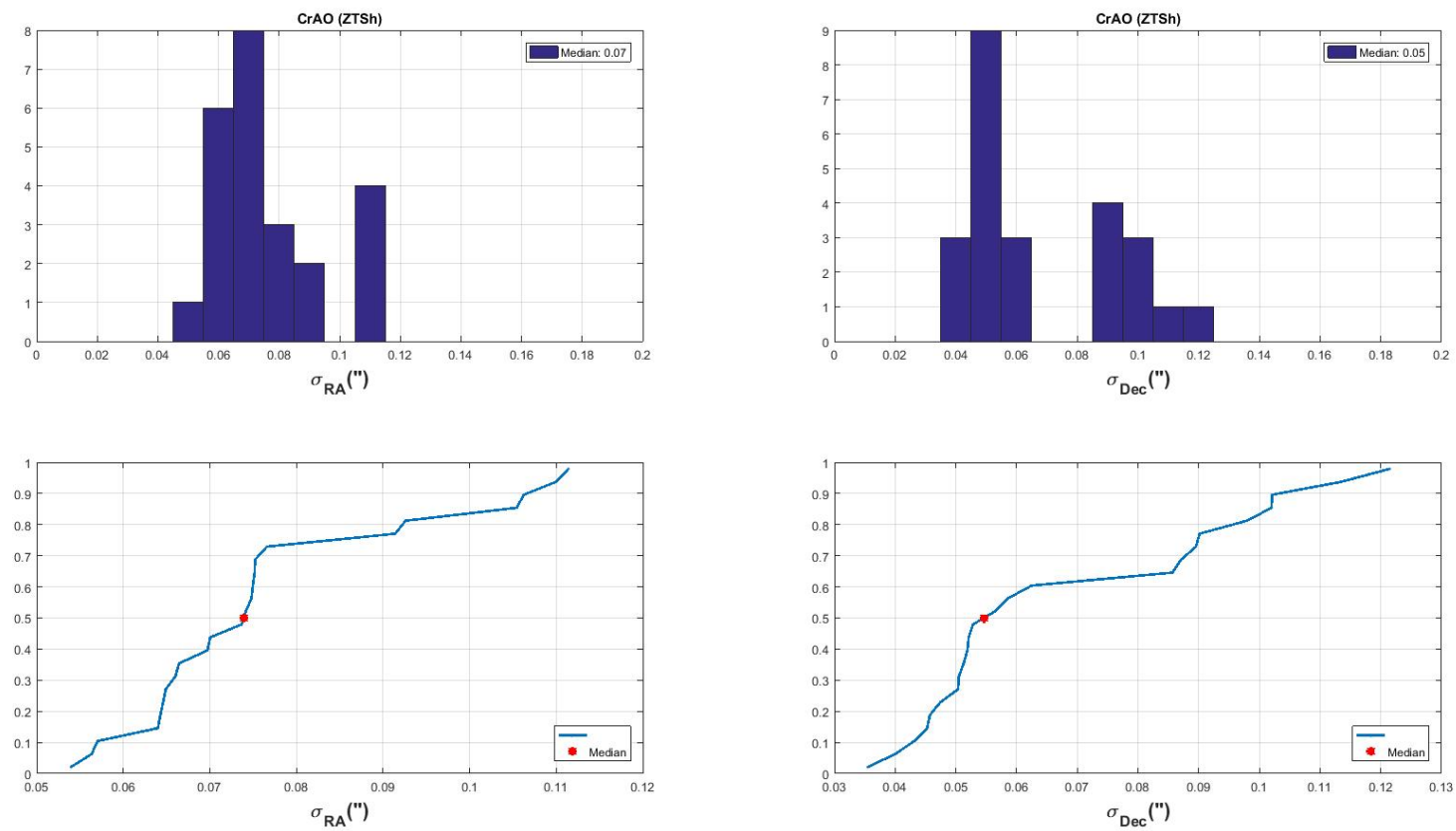

Рис. 6. Гистограммы распределения CKO невязок по RA и Decl (верхний ряд) и кумулятивная функция (нижний ряд). Красная точка соответствует медианному значению

Сразу после запуска были начаты наблюдения на телескопе АТ-64 и продолжены на АЗТ-11 и ЗТШ с целью контроля движения аппарата и коррекции его орбиты. В течение 75 ночей проведено более 86 сетов наблюдений $(\sim 3000$ положений $)$, которые продолжаются до сих пор. Типичные среднеквадратичные ошибки положения составляют: для АТ-64 - 0.23", для АЗТ-11 - 0.15" и для ЗТШ - 0.07" (рис. 6).

Разгонный блок “ДМ-03", в отличие от "Спектр-РГ", вышел на гелиоцентрическую орбиту. По нему также проводились наблюдения до прекращения видимости. Последние его наблюдения проведены на ЗТШ 27 ноября 2019 года на расстоянии 4 млн км как объект $21^{m}$.

\section{Mars-2020 (2020-052A)}

Для исследования Марса 30 июля 2020 года в США был запущен KA Mars-2020 с марсоходом Perseverance. Его наблюдения на отлетной траектории к Марсу начались 30 июля на AT-64 и продолжились 14, 15 и 19 августа на ЗТШ.

Цель наблюдений состояла в практическом определении предельных возможностей оптических позиционных наблюдений на максимальных дистанциях.

На АТ-64 были получены 4 сета (129 положений, общий интервал наблюдения 3 часа), при яркости объекта $14.5-14.8^{m}$ невязки положения составляли $0.15-0.23^{\prime \prime}$. На тот момент расстояние до объекта составляло 0.001 а.е. ( 150 тыс. км).

Таблица 2.

\begin{tabular}{ccccccc}
\hline Date & Time, UT & \#pos & $\begin{array}{c}\text { Duration } \\
(\min )\end{array}$ & Mag & std pos (arcsec) & $\begin{array}{c}\text { Range } \\
\left(10^{6} \mathrm{~km}\right)\end{array}$ \\
20200814 & $23: 49-00: 02$ & 4 & 12.5 & 21.2 & 0.090 .06 & 5.18 \\
20200815 & $00: 49-01: 21$ & 13 & 31.7 & 21.2 & 0.170 .27 & 5.20 \\
20200815 & $21: 53-22: 04$ & 4 & 10.5 & 21.6 & 0.020 .01 & 5.48 \\
20200819 & $00: 40-00: 55$ & 7 & 14.7 & 21.8 & 0.230 .12 & 6.50 \\
\hline
\end{tabular}

Спустя 2 недели начались наблюдения на ЗТШ. В течение трех ночей 14, 15 и 19 августа 2020 г. были получены 4 сета наблюдений (всего 28 положений, таблица 2). 
В это время KA Mars-2020 находился на удалении более 5 млн км. Наблюдения проводились с экспозицией 2 мин., яркость объекта была слабее $21^{m}$. Скорости видимого движения составляли $1^{\prime \prime}$ /мин. и менее. Последние наблюдения, проведенные 19 августа в 00:50 UT, получены на удалении 6.5 млн км. Объект был $21.8^{m}$ при фазовом угле 51 градус. Точность положения составила 0.1-0.2" .

\section{3 Заключение}

Работа на ЗТШ по космическому мусору показала, что данный телескоп является “машиной открытий".

ЗТШ позволил каталогизировать объекты в ГСО размером порядка 10 см. Время, затрачиваемое на поиск нового объекта, составляло около 10 минут. Эти объекты, как правило, имели большие отношения площади к массе, что требовало регулярного контроля их траектории.

Фотометрические наблюдения избранных объектов показали сильную переменность, перепад блеска составлял несколько звездных величин, а в некоторых случаях превосходил $5^{m}$, что говорит о "неправильной" форме таких объектов. Скорее всего, это могут быть плоские элементы конструкций КА или фрагменты экранно-вакуумной изоляции. Астрометрическая точность измерений объектов $18-20^{m}$ составляла $0.2-0.5^{\prime \prime}$.

Однако загруженность ЗТШ другими астрофизическими задачами, незначительное количество времени, выделяемого под данную задачу, и отсутствие соответствующей оптической поддержки привели к завершению нами работ по космическому мусору на этом телескопе.

Наблюдения далеких КО актуальны не только для контроля траектории (независимо от радиотехнических средств), но и для распознавания искусственных объектов среди естественных (Buzzoni et al., 2019). Действительно, в последнее время все больше открывается астероидов, сближающихся с Землей. Контроль этой области пространства в последние два десятилетия сильно активизировался. Ежегодно открывается 1-2 тыс. таких объектов. Среди них обнаруживаются искусственные объекты - отработавшие ступени ракет, межпланетные аппараты, запущенные в 60-70-х годах. Например, объект $2010 \mathrm{KQ}$, вероятно, является отработавшей четвертой ступенью ракеты-носителя станции “Луна-23" (Miles, 2011; Yeomans et al., 2010). В 2020 г. Центр малых планет зарегистрировал объект с предварительным номером 2020 SO. После дальнейших исследований им оказалась верхняя ступень ракеты-носителя - Centaur, которая использовалась для доставки спускаемого аппарата Surveyor 2 на Луну в 1966 году (O’Neill, Handal, 2020).

Кроме этого, еще ряд объектов, обнаруженных при астероидных обзорах неба, по-видимому, имеют искусственное происхождение. Джонатан Макдауэлл (Jonathan McDowell) из ГарвардскоСмитсоновского центра астрофизики отметил, что было "много, много неловких инцидентов, когда объекты на далекой орбите ... получали предварительные обозначения астероидов за несколько дней до того, как стало понятно, что они были искусственными" (Dunn, 2020).

Понятно, что из-за малости размеров ступеней и аппаратов, а также принимая во внимание большие расстояния до объектов, находящихся на гелиоцентрических орбитах, наблюдать их в течение большого интервала времени не получится. Единственное, что остается, это вести наблюдения далеких КО на максимально возможном интервале. Было бы весьма желательно также организовать их мониторинг, аналогичный мониторингу АC3.

\section{Литература}

Захваткин М., 2015. Частное сообщение. [Zahvatkin M., 2015. Private message.]

Прокофьева-Михайловская В.В., 2008. Изв. Крымск. Астрофиз. Обсерв. Т. 104. № 5. С. $126-131$.

[Prokofjeva-Mikhailovskaja V., 2008. Izv. Krymsk. Astrofiz. Observ., vol. 104, no. 5, pp. 126-131. (In Russ.)]

Agapov V., Dick J., Guseva I., et al., 2005a. In Danesy D. (Ed.), Proceedings of the Fourth European Conference on Space Debris, Darmstadt, Germany. ESA Special Publication, vol. 587, p. 119.

Agapov V., Biryukov V., Kiladze R., et al., 2005b. In Danesy D. (Ed.), Proceedings of the Fourth European

Conference on Space Debris, Darmstadt, Germany. ESA Special Publication, vol. 587, p. 153.

Buzzoni A., Altavilla G., Fan S., et al., 2019. Advances in Space Research. vol. 63, no. 1, pp. 371-393. 
Dunn M., 2020. Fake asteroid? NASA expert IDs mystery object as old rocket. Associated Press, October 11, 2020. Available at: https://apnews.com/article/ science-us-news-ap-top-news-asteroids-moon-5a3d5465b53b09101bd47c3bc8cac84e (Accessed: 09.09.2021).

Klinkrad H., Alby F., Alwes D., et al., 2005. In Danesy D. (Ed.), Proceedings of the Fourth European Conference on Space Debris, Darmstadt, Germany. ESA Special Publication, vol. 587, p. 25.

Miles R. 2011. J. Br. Astron. Assoc., vol. 121, no. 6, pp. 350-354.

O'Neill I.J., Handal J., 2020. Earth May Have Captured a 1960s-Era Rocket Booster. Nov. 12, 2020. Available at: www.nasa.gov/feature/jpl/ earth-may-have-captured-a-1960s-era-rocket-booster, (Accessed: 09.09.2021).

Seitzer P., Jorgensen K., Africano J.L., et al., 2005. Proceedings of the AMOS technical conference, September 5-9, Maui, Hawaii, pp. 224-229.

Schildknecht T., Musci R., Ploner M., et al., 2001. In Huguette Sawaya-Lacoste (Ed.), Proceedings of the Third European Conference on Space Debris, 19-21 March 2001, Darmstadt, Germany. Noordwijk, Netherlands: ESA Publications Division vol. 1, pp. 89-93.

Schildknecht T., Musci R., M., Flury W., et al. 2005. In Danesy D. (Ed.), Proceedings of the Fourth European Conference on Space Debris. ESA Publications Division, Noordwijk, The Netherlands, vol. 587, pp. 113-118.

Sochilina A.S., Grigoriev K.V., Vershkov A.N., et al., 2001. In Huguette Sawaya-Lacoste (Ed.), Proceedings of the Third European Conference on Space Debris, 19-21 March 2001, Darmstadt, Germany. Noordwijk, Netherlands: ESA Publications Division, vol. 1, pp. 367-372.

Sochilina A., Kiladze R., Grigoriev K., et al. 2002. Adv. Space Res., vol. 34, iss. 5, pp. 1198-1202.

Technical Report on Space Debris, 1999. Technical report on space debris: text of the report adopted by the Scientific and Technical Subcommittee of the United Nations Committee on the Peaceful Uses of Outer Space. New York: United Nations.

Yeomans D., Chodas P., Chesley S., 2010. Asteroid 2010 KQ: Probably a Rocket Body. May 27, 2010. Available at: https://cneos.jpl.nasa.gov/news/news168.html, (Accessed: 23.09.2021). 


\title{
Observations of artificial space objects with the $2.6 \mathrm{~m}$ Shajn Telescope at the Crimean Astrophysical Observatory
}

\author{
V.V. Rumyantsev, V.V. Biryukov \\ Crimean Astrophysical Observatory, Nauchny 298409 \\ rum@craocrimea.ru
}

\begin{abstract}
The paper provides an overview of studies of artificial objects in the near-earth space, which have been carried out with the Shajn telescope since 2005. One of the study objectives was to develop a technique for observing and cataloguing small objects $(\sim 10-25 \mathrm{~cm})$ in the geostationary orbit with a brightness of $18-20^{m}$. Despite the strong limitation of observational time, the use of Shajn telescope for solving this problem was quite effective. It is shown that it is possible to detect and catalog small-sized objects in the geostationary orbit even with a telescope with a small field of view ( 8-12 arcmin).

A new modern device (panoramic CCD photometer) was created and placed at the prime focus of the Shajn telescope to solve tasks of searching for weak, highly variable, "dynamic" objects.

In the period from 2011 to 2021, the Shajn telescope carried out observations of distant spacecrafts Spektr-R, Gaia, Spektr-RG, and Mars-2020. Particular attention was paid to the Russian astrophysical observatory Spektr-RG in the halocentric orbit around the L2 Lagrange point of the Sun - Earth system. Observations of this scientific spacecraft continue to this day. The accuracy of the obtained astrometric estimates is such that the median values of RMS errors of position are $0.055^{\prime \prime}$ and $0.075^{\prime \prime}$ to RA and Decl, respectively. The spacecraft Mars-2020 on the flight trajectory to Mars was observed at a distance of up to 6.5 million $\mathrm{km}$ as an object of $21.8^{\mathrm{m}}$.

The task of observing distant spacecrafts remains relevant for recognizing artificial objects among the numerous natural ones detected in the near-earth space.
\end{abstract}

Key words: near-earth space, geostationary orbit, artificial space objects, small space debris, distant spacecrafts 